\title{
Helium in polygonal nanopores at zero temperature: Density functional theory calculations
}

\author{
A. Hernando, ${ }^{1}$ E. S. Hernández, ${ }^{2}$ R. Mayol, ${ }^{1}$ and M. $\mathrm{Pi}^{1}$ \\ ${ }^{1}$ Departament d'Estructura i Components de la Matèria, Facultat de Física, \\ and IN2 UB, Universitat de Barcelona, 08028 Barcelona, Spain \\ ${ }^{2}$ Departamento de Física, Facultad de Ciencias Exactas y Naturales, Universidad de Buenos Aires \\ and Consejo Nacional de Investigaciones Científicas y Técnicas, 1428 Buenos Aires, Argentina \\ (Received 23 November 2007; revised manuscript received 10 April 2008; published 22 May 2008)
}

\begin{abstract}
We investigate adsorption of helium in nanoscopic polygonal pores at zero temperature using a finite-range density functional theory. The adsorption potential is computed by means of a technique denoted as the elementary source method. We analyze a rhombic pore with Cs walls, where we show the existence of multiple interfacial configurations at some linear densities, which correspond to metastable states. Shape transitions and hysterectic loops appear in patterns which are richer and more complex than in a cylindrical tube with the same transverse area.
\end{abstract}

DOI: 10.1103/PhysRevB.77.195431

PACS number(s): 68.08.Bc, 68.65.-k, 68.35.Np

A widely investigated topic in physics of quantum fluids is the wetting behavior of helium on substrates of different adsorbing powers. For flat surfaces, the latter is determined by the adatom-adsorber interaction; however, the geometrical structure of a matter exposed to the vapor modifies the adsorption strength and the growth of the film. ${ }^{1} \mathrm{~A}$ special concern is the filling of pores. Most reported research in this field addresses classical fluids at or close to bulk coexistence and resorts to Monte Carlo, molecular dynamics, and meanfield approaches..$^{2-7}$ These systems display a rich variety of behaviors, and we expect that this is also true for liquid ${ }^{4} \mathrm{He}$ in extremely cold quantum regime. In addition to the competition between adhesive (fluid-wall) and cohesive (fluidfluid) forces, which in wetting problems gives rise to phase changes governed by surface effects, pores make room to interplay among finite sizes, geometrical shape of the confinement, and varying dimensionality. Metastable fluid states show up in hysteresis loops in the sorption isotherms, traditionally associated with the onset of capillary condensation (CC) ${ }^{8}$ Furthermore, as pointed out in Ref. 3, among the complications arising from almost every model of condensation in pores, there are uncertainties in both the substratefluid and the fluid-fluid interactions. The latter is commonly selected as that in the bulk, while for the adhesive forces, the simplest reduction is the summation of Lennard-Jones pair interactions, ${ }^{9}$ which ignores effects that are associated with the polarization and three-body forces.

A simple matter unit out of which one may construct polygonal pores of various shapes is the infinite linear wedge. Recently, we presented the theoretical study of condensation of superfluid ${ }^{4} \mathrm{He}$ in wedges, ${ }^{10}$ employing a zero-temperature, finite-range density functional (FRDF) that has proven helpful to understand a large variety of phenomena in finite systems of liquid helium isotopes and their mixtures. ${ }^{11}$ The summation employed there to construct the adsorption potential of two semi-infinite walls meeting at a corner can be improved by a newly reported method ${ }^{12}$ that gives the potential of a substrate of arbitrary shape, provided that the $a b$ initio adsorption field for the semi-infinite material with a planar surface is known. This method consists of solving an inverse problem to determine the elementary source potential that gives rise to the planar adsorption field and allows the construction of potentials for curved matter, for polygonal pores, and for rough surfaces in a relatively simple way and at a rather low numerical cost.

In this work, we investigate adsorption of helium in nanoscopic polygonal pores at zero temperature employing the FRDF of Ref. 13 plus an adsorption potential built by the method of Ref. 12. We analyze a rhombic pore, for which a rich collection of interfacial configurations and shape transitions appear along the full path from adsorption of quasione-dimensional samples of helium up to CC. In particular, we show that different metastable states with grand potential above the equilibrium value may be reached at a given fluid density, according to the choice of the initial condition. This is well known in theory and experiments of classical fluids in pores already discussed at length in the literature. ${ }^{3}$

Rhombic pores appear in mineral crystals and are manufactured in metallic and organic materials since the 1960s. ${ }^{14}$ The wetting and filling behavior of such pores with Cs walls is an interesting issue, since the "heliophobicity" of planar Cs at low temperatures is a consequence of the weak interaction between $\mathrm{Cs}$ and $\mathrm{He}$ atoms as compared to the cohesive $\mathrm{He}-\mathrm{He}$ forces; however, the wall-adatom interaction can be tuned by the convexity of the pore walls and may drive the substrate into a "heliophilic" regime. Our investigation of the shapes of helium samples ${ }^{10}$ suggests that for some combination of rhombus angles, it might be possible to have a concave or flat meniscus at the narrow corner, which hosts the absolute minimum of the external potential, coexisting with a convex meniscus at the neighboring vertex.

We performed FRDF computations of helium density in a Cs rhombic pore of $60^{\circ}$ and side equal to $50 \AA$. This large size has been chosen in order to visualize better the effects of the angles and of the flat walls on the growth pattern of the adsorbed film, as discussed below; moreover, microrhombic pores of this shape have been fabricated, i.e., out of tracketched mica. ${ }^{15}$ The calculational details to compute the helium density are the same as in previous literature. ${ }^{10,11}$ The potential along the diagonals of this pore obtained by the elementary source method ${ }^{12}$ is shown in Fig. 1, together with that for a cylindrical tube with the same transverse area. As 


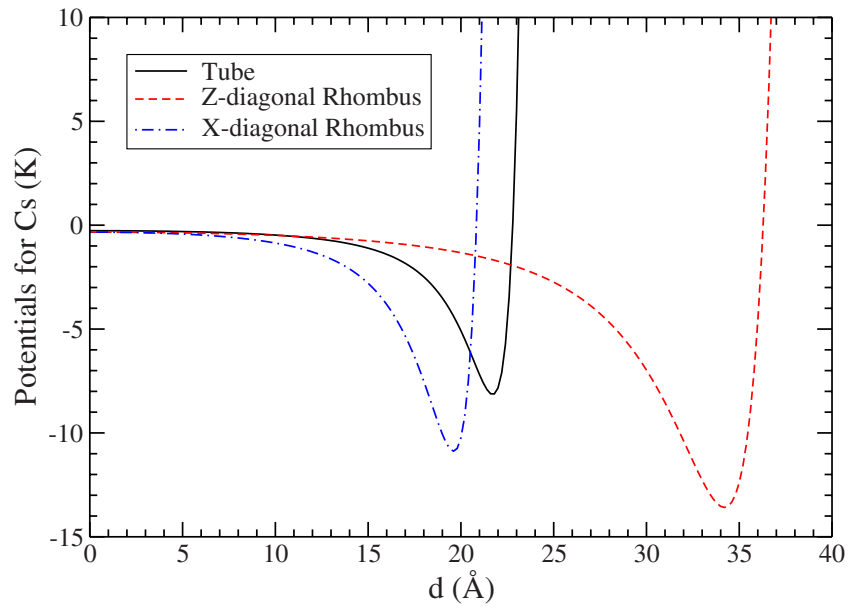

FIG. 1. (Color online) Integrated potentials (Ref. 12) along the diagonals of a rhombic Cs pore with $50 \AA$ side and $60^{\circ}$ on the $z$ axis and for a Cs cylinder with equal area, with distances measured from the center of the pore. The respective lengths are 25 and 43.30 $\AA$ for the half-diagonals and $26.25 \AA$ for the cylinder radius.

expected, ${ }^{10}$ the presence of angles substantially enhances the attraction with respect to a curved smooth surface.

Our recent investigation of $\mathrm{CC}$ and the formation of bridges between two parallel plates ${ }^{16}$ showed that two or more equilibrium configurations, which are connected by a hysteresis loop, appear within a range of coverage. This multiplicity is intrinsic to any method of solution of a nonlinear equation ${ }^{6}$ where an initial density profile is driven toward equilibrium, for instance, by the imaginary time method here employed. ${ }^{10,11}$ For classical fluids in pores, density functional calculations provide metastable solutions arranged into hysterectic loops, which are compatible with experiment and with descriptions based on Monte Carlo techniques for pores wider than a few angstrom. $3,4,6$

Due to the presence of corners at different angles, which host local minima in the external potential, we should expect multiple solutions at the same $n$, some corresponding to metastable physical states. We have encountered different equilibrium solutions by starting the imaginary time evolution, for a given linear density $n$, from various initial configurations. We have performed at least two different runs for each linear density, starting from helium filling homogeneously (i) the whole pore and (ii) only a single $60^{\circ}$ wedge up to the shortest diagonal.

In Fig. 2, we show the grand potential per particle, with full and dashed-dotted lines corresponding to initial condition (i) and (ii), respectively. The adsorption isotherms $\mu(n)$ are shown in Fig. 3. In view of the complexity of these figures, we examine the trend of $\Omega / N$ and relate the distinct features to those in $\mu(n)$. For the lowest linear densities, pattern (a) on path (ii) is the stable configuration corresponding to the absolute minimum of the grand potential. Condensation $(\Omega=0)$ takes place on this trajectory near $n_{c}^{(i i)}$ $=3 \AA^{-1}$ at $\mu_{c}$ around $-7.75 \mathrm{~K}$, which is well below the bulk coexistence figure of $-7.15 \mathrm{~K}$. Instead, along trajectory (i), condensation takes place around $n_{c}^{(i)}=5.8 \AA^{-1}$ at essentially the same chemical potential. At a linear density around $10 \AA^{-1}$, path (i) overcomes (ii), which in turn destabilizes,

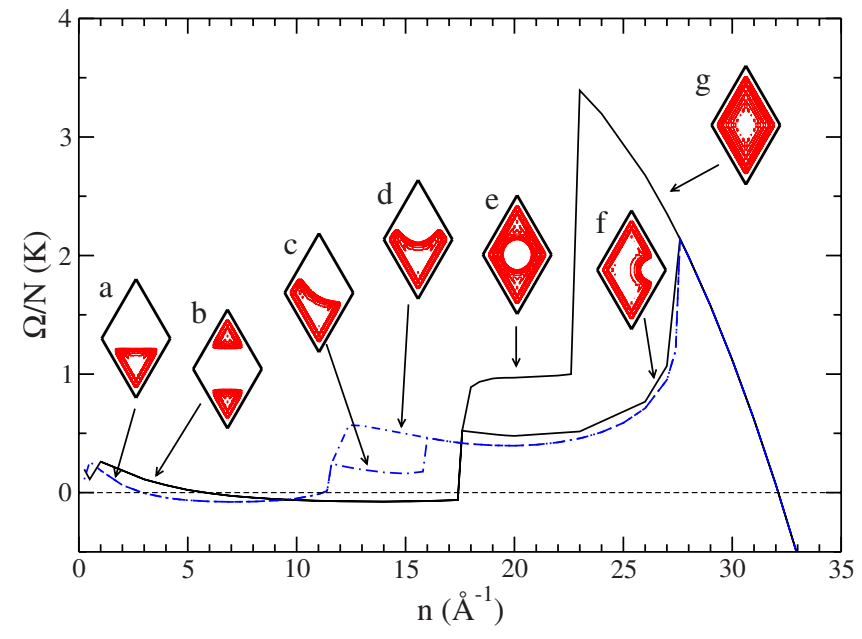

FIG. 2. (Color online) Grand potential per particle $\Omega / N$ along paths (i) and (ii) (full and dashed-dotted lines, respectively) in the rhombic Cs adsorbing potential of Fig. 1. The insets (a)-(g) have been, respectively, drawn for linear densities $n=9,9,12,16,20,26$, and $29 \AA^{-1}$. The arrows point to the path (not to the value of $n$ ).

i.e., $\Omega$ becomes positive with negative $d \mu / d n$, for $n$ slightly above $11 \AA^{-1}$.

The unstable trajectory (ii) evolves further by filling adjacent corners with a hysterecticlike loop that corresponds (c) to an asymmetric and (d) to a symmetric landscape, the former possessing a smaller grand potential, however, positive. This loop has been induced by adding some numerical noise at the start so as to break the symmetry with respect to the longest diagonal as in pattern (c). The stable path (i) destabilizes near $17.5 \AA^{-1}$ and there also appears a hysterecticlike loop produced by symmetry-breaking noise. In either case, both the symmetric and the asymmetric fillings are preserved along the imaginary-time evolution, and the latter always presents the lowest positive grand potential. For $n$ $\geq 28 \AA^{-1}$, both trajectories merge and yield a unique pattern (g) that becomes stable at $n_{\mathrm{CC}}=32.5 \AA^{-1}$ for a chemical potential $\mu_{\mathrm{CC}}$ around $-8.5 \mathrm{~K}$, which should be regarded as the first order phase transition to CC. At $34 \AA^{-1}$, the chemical

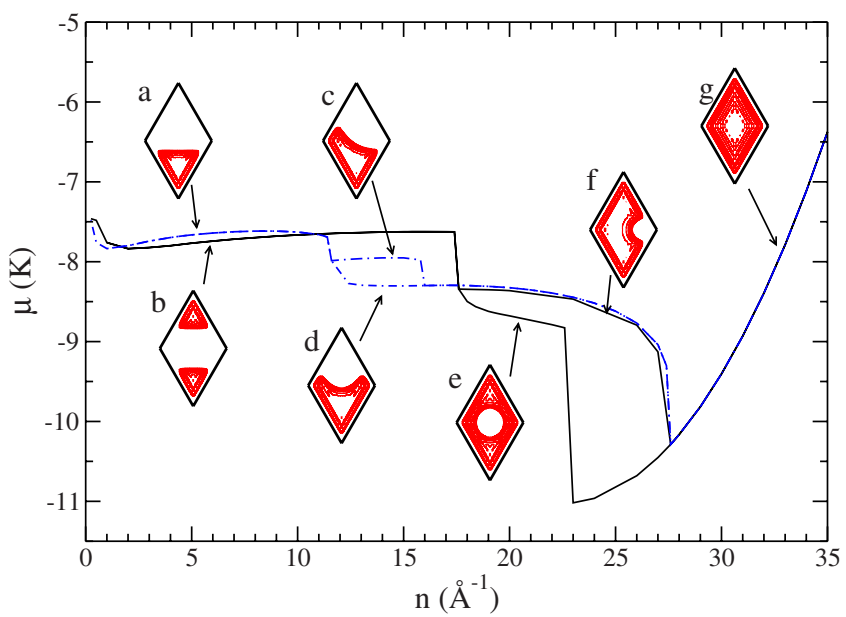

FIG. 3. (Color online) Same as Fig. 2 for the chemical potential $\mu(n)$. 


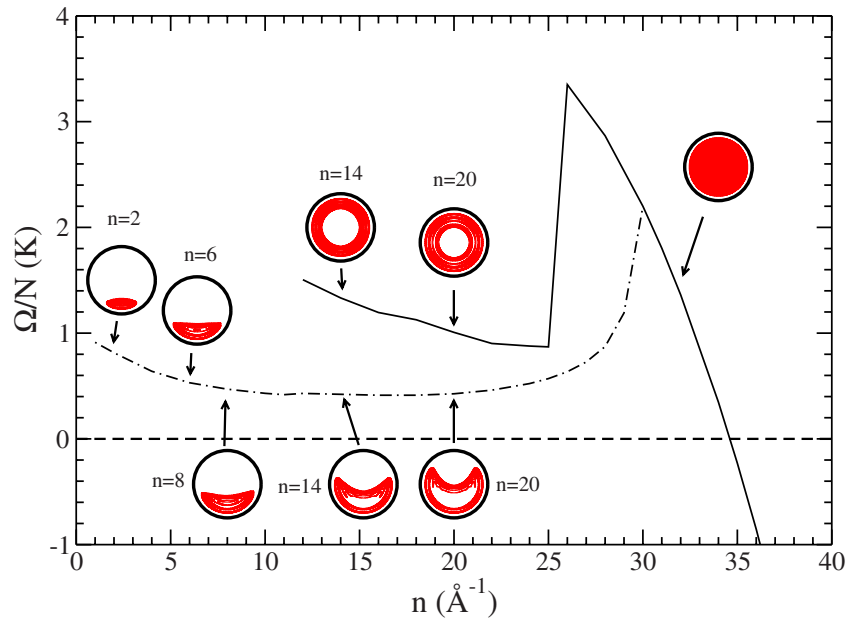

FIG. 4. (Color online) Same as Fig. 2 for a cylindrical pore with same area as the $50 \AA$, rhombus.

potential crosses the bulk value and grows with a large derivative, signaling the end of validity of the current calculation.

We recognize two regions in Figs. 2 and 3. Below $n$ $\approx 17 \AA^{-1}$, the isotherm reveals sequential adsorption by filling the corners, a process that constitutes the first stage in the occupation of a pore ${ }^{3}$ and replaces, for this geometry, the well-known "layering" of a film on planar surfaces. Although in the density range between 17.5 and $32.5 \AA^{-1}$ the system is unstable against density fluctuations, the FRDF method always gives rise to continuous trajectories, which represent possible branches of the equation of state of the helium atoms. The higher $n$ region above $17 \AA^{-1}$ corresponds to CC and the loop resembles the adsorption-desorption hysteresis cycle described in the literature, ${ }^{3,6}$ with an upper adsorptionlike branch governed by adhesion to the pore walls and a lower desorptionlike path where symmetry-breaking bubbles may form. Each isotherm in Fig. 3 displays two van der Waals loops; one at low density ending at $n_{c}$; the second ending at the CC threshold $n_{\mathrm{cc}}$. Closer examinations of the curves indicate that the origin of the latter Maxwell construction takes place around $n=17 \AA^{-1}$, where paths (i) and (ii)

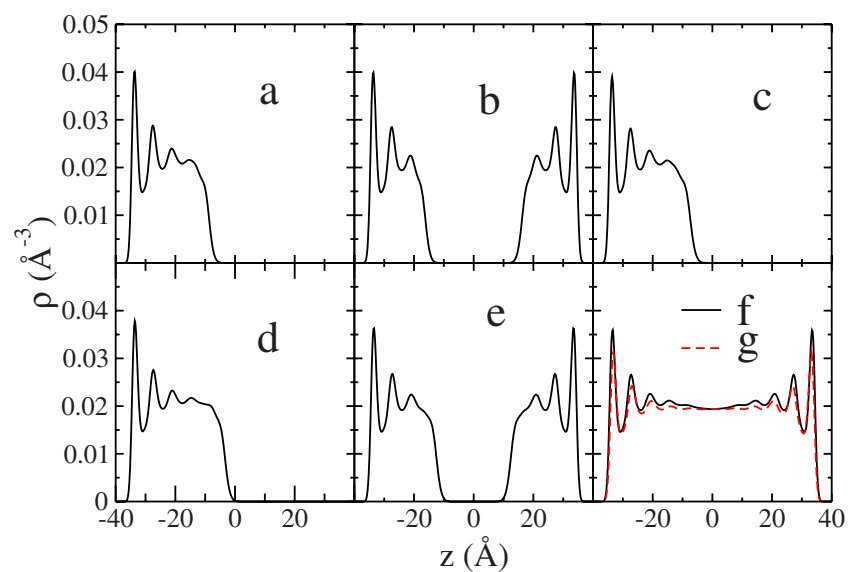

FIG. 5. (Color online) Density $\rho(0, z)$ in the rhombic pore of Figs. 2 and 3.

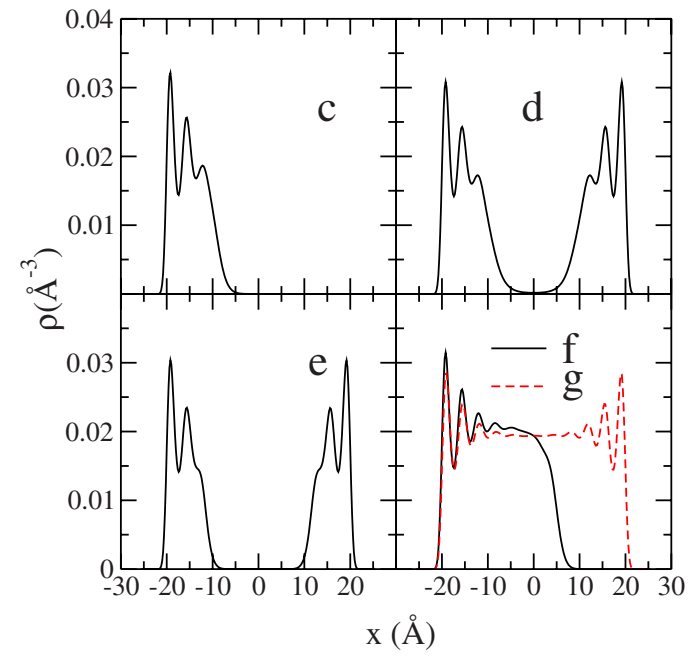

FIG. 6. (Color online) Density $\rho(x, 0)$ in the rhombic pore of Figs. 2 and 3.

cross. Note that the patterns (e) and (g) at the edges of this plateau, respectively, represent a low-density phase with fluid sticking to the walls, which corresponds to stable multilayer adsorption and the fully condensed state at high density (cf. Figs. 5 and 6). These are the phases coexisting at $\mathrm{CC},{ }^{5}$ which are unambiguously visualized in Monte Carlo simulations. ${ }^{5,7}$

The CC loop is similar to the one in Fig. 4 for a cylindrical pore of radius $R \approx 26.25 \AA$, with the same area as the

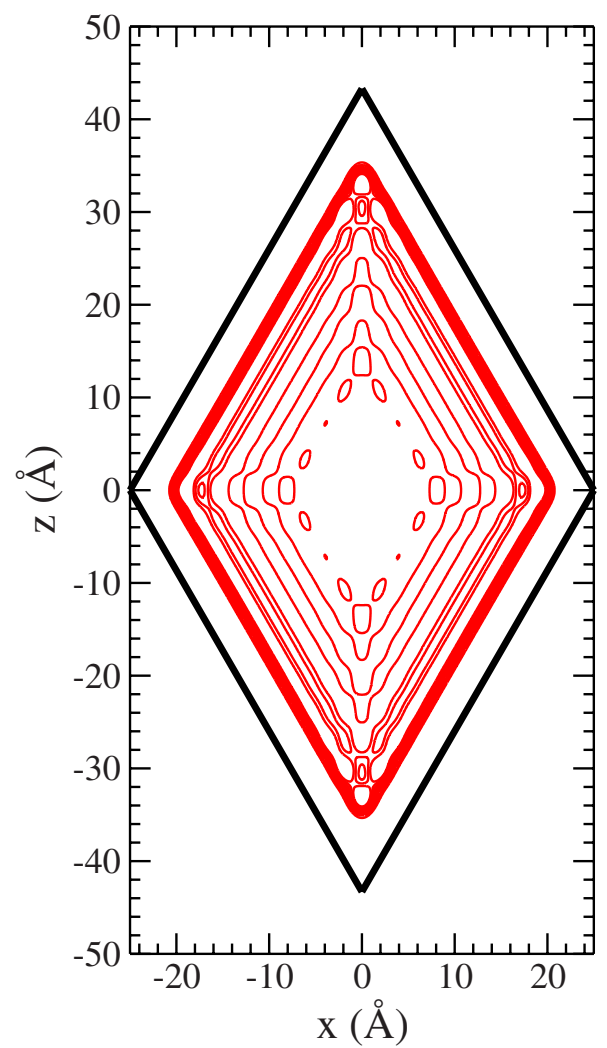

FIG. 7. (Color online) Density contours of type (g) for $n$ $=29 \AA^{-1}$. 
$50 \AA$ A rhombus. As in Fig. 2, the full and dashed-dotted lines, respectively, contain patterns evolved from helium filling homogeneously (i) the whole cylinder and (ii) a half tube. Although we could not reach converging solutions along path (i) for densities below $12 \AA^{-1}$, it is clear that both curves must join the origin of coordinates smoothly after reaching their maxima at low densities. The main qualitative difference between Figs. 2 and 4 is the disappearance of the branches associated with filling of the corners. Moreover, the vanishing of the grand potential near $34.5 \AA^{-1}$ for $\mu$ around $-8.2 \mathrm{~K}$ is an indication that a radial configuration of helium wets the tube walls, which is in agreement with earlier findings for Cs cylinders. ${ }^{17}$ For this radius, adhesion to the cylindrical walls is not sufficient to secure condensation at lower densities. This is a prelude for the formation of finite size drops or bubbles inside the tube, as obtained for planar geometries. ${ }^{18}$

Figures 5 and 6 , respectively, show the densities $\rho(0, z)$ and $\rho(x, 0)$ along the long ( $z$ axis) and short ( $x$ axis) diagonals. In panels (a) and (b) of Fig. 5 the peaks represent quasione-dimensional helium accumulating near the vertices, according to the typical growth pattern in a wedge. ${ }^{16}$ Panels (c) to (e) illustrate the various forms of filling the four corners and we note the symmetry-breaking effect in panels (d), where helium is capillary condensed along the shortest diagonal (Fig. 6) while keeping empty one narrow corner (Fig. 5). A similar effect takes place in panels (f) exchanging diagonals.

The lowest right panels confirm that in configuration $(\mathrm{g})$, helium is capillary condensed. The density contours displayed in more detail in Fig. 7 illustrate the competition between the "spaghettilike" growth by an accumulation of quasi-one-dimensional matter, and the "lasagnalike" pattern, which is characteristic of planar film thickening by addition of quasi-two-dimensional layers. This mechanism can only occur in pores with sufficiently long sides.

As a final remark, we comment that since the universe of initial conditions is overwhelmingly large, the present work is far from exhaustive and serves the purpose of illustrating the kind of scenarios that may appear.

We are grateful to Manuel Barranco for enlightening discussions. This work has been performed under Grants No. FIS2005-01414 from DGI, Spain (FEDER), Grant No. 2005 SGR00343 from Generalitat de Catalunya and in Argentina, Grant No. PIP 5138/05 from CONICET, Grant No. PICT 31980/05 from ANPCT, and Grant No. X298 from University of Buenos Aires.
${ }^{1}$ C. Rascón and A. O. Parry, J. Chem. Phys. 123, 024798 (2000); L. Bruschi, A. Carlin, and G. Mistura, Phys. Rev. Lett. 89, 166101 (2002); C. Rascón and A. O. Parry, Nature (London) 407, 986 (2003); G. McHale, N. J. Shirtcliffe, S. Aqil, C. C. Perry, and M. I. Newton, Phys. Rev. Lett. 93, 036102 (2004); O. Gang, Kyle J. Alvine, Masafumi Fukuto, Peter S. Pershan, Charles T. Black, and Benjamin M. Ocko, ibid. 95, 217801 (2005); L. Bruschi, G. Fois, G. Mistura, M. Tormen, V. Garbin, E. di Fabrizzio, A. Gerardino, and M. Natali, J. Chem. Phys. 125, 144709 (2006); M. Tasinkevych and S. Dietrich, Phys. Rev. Lett. 97, 106102 (2006).

${ }^{2}$ R. Evans, U. Marini Bettolo Marconi, and P. Tarazona, J. Chem. Soc., Faraday Trans. 2 82, 1763 (1986); P. C. Ball and R. Evans, Langmuir 5, 714 (1989).

${ }^{3}$ L. D. Gelb, K. E. Gubbins, R. Radhandakrishnan, and M. Sliwinska-Bartkowiak, Rep. Prog. Phys. 62, 1573 (1999).

${ }^{4}$ A. V. Neimark, P. I. Ravikovitch, and A. Vishnyakov, Phys. Rev. E 62, R1493 (2000); P. I. Ravikovitch, A. Vishnyakov, and A. V. Neimark, ibid. 64, 011602 (2001).

${ }^{5}$ L. D. Gelb, Mol. Phys. 100, 2049 (2002).

${ }^{6}$ A. Neimark, P. I. Ravikovich, and A. Vishnakov, J. Phys.: Condens. Matter 15, 347 (2003).

${ }^{7}$ R. Paul and H. Rieger, J. Chem. Phys. 123, 024708 (2005).

${ }^{8}$ L. H. Cohan, J. Am. Chem. Soc. 60, 433 (1938).
${ }^{9}$ M. W. Cole, F. Ancilotto, and S. M. Gatica, J. Low Temp. Phys. 138, 195 (2005); M. Rossi, D. E. Galli, and L. Reatto, ibid. 146, 95 (2007).

${ }^{10}$ E. S. Hernández, F. Ancilotto, M. Barranco, R. Mayol, and M. Pi, Phys. Rev. B 73, 245406 (2006).

${ }^{11}$ M. Barranco, R. Guardiola, S. Hernández, R. Mayol, J. Navarro, and M. Pi, J. Low Temp. Phys. 142, 1 (2006).

${ }^{12}$ A. Hernando, E. S. Hernández, R. Mayol, and M. Pi, Phys. Rev. B 76, 115429 (2007).

${ }^{13}$ F. Dalfovo, A. Lastri, L. Pricaupenko, S. Stringari, and J. Treiner, Phys. Rev. B 52, 1193 (1995).

${ }^{14}$ M. A. M. Beerlage, J. M. M. Peeters, J. A. M. Nolten, M. H. V. Mulder, and H. Strathmann, J. Appl. Polym. Sci. 75, 1180 (2000); R. G. Harrison, N. K. Dally, and A. Y. Nazarenko, Chem. Commun. (Cambridge) 2000, 1387; S. Matthias, F. Müller, and U. Gösele, J. Appl. Phys. 98, 023524 (2005).

${ }^{15}$ P. F. Mc Kenzie, R. M. Webber, and J. L. Anderson, Langmuir 10, 1539 (1994).

${ }^{16}$ R. Mayol, F. Ancilotto, M. Barranco, E. S. Hernández, and M. Pi, J. Low Temp. Phys. 148, 851 (2007).

${ }^{17}$ L. Szybisz and S. M. Gatica, Phys. Rev. B 64, 224523 (2001).

${ }^{18}$ M. Barranco, M. Guilleumas, E. S. Hernández, R. Mayol, M. Pi, and L. Szybisz, Phys. Rev. B 68, 024515 (2003). 